\title{
Explorative Analysis of 60 Metagenomes to Unveil the Abundance of Widespread Functions in the Environment and Its Weight on Functional Indices
}

Robert Starke ( $\sim$ robert.starke@biomed.cas.cz)

Mikrobiologicky ustav Akademie ved Ceske republiky https://orcid.org/0000-0003-3020-4605

Sarah Piché-Choquette

Mikrobiologicky ustav Akademie ved Ceske republiky

\section{Research}

Keywords: bacterial and fungal functional richness, metagenome functionality, Diversity, aquatic and terrestrial metagenomes

Posted Date: August 2nd, 2020

DOl: https://doi.org/10.21203/rs.3.rs-50009/v1

License: (c) (1) This work is licensed under a Creative Commons Attribution 4.0 International License.

Read Full License 
Title: Explorative analysis of 60 metagenomes to unveil the abundance of widespread functions in the environment and its weight on functional indices

Running title: The buffering effect of widespread functions

Authors: Robert Starke* (robert.starke@biomed.cas.cz) and Sarah Piché-Choquette (sarah.piche@biomed.cas.cz)

Affiliation: Laboratory of Environmental Microbiology, Institute of Microbiology of the Czech Academy of Sciences, Prague, Czech Republic

*Corresponding author 


\begin{abstract}
Background: The total bacterial and fungal functional richness was recently predicted to be millions of KEGG level 3 functions but due to limited space and resource availability together with constraining environmental conditions, the local functionality only comprises a subset of the total functionality. However, widespread functions whose abundance and redundancy depends on species richness or sequencing depth could bias functional indices. Here, we used 20 randomly chosen extant metagenomes from low to high species richness each from aerial, aquatic and terrestrial ecosystems to quantify the distribution of widespread KEGG level 3 functions and their buffering effect on diversity, redundancy and richness.

Results: A different number of widespread functions were detected in each ecosystem with 1,201 found in all three. Diversity found to be similar in the three ecosystems and redundancy that was significantly lower in aerial metagenomes showed identical patterns when all functions were used or when the widespread functions were removed. However, functional richness changed from being significantly higher in aquatic than in terrestrial and aerial metagenomes when all functions are used to no significant differences when the widespread functions are removed. The variance in diversity and richness was higher without widespread functions, making it possible to compare highly dense environments that would otherwise result in similar values.
\end{abstract}

Conclusions: Taken together, we describe the widespread functiome in aerial, aquatic and terrestrial metagenomes, and its buffering effect on functional indices that are driven by widespread functions which is why, moving forward, we recommend to remove widespread functions from the analysis of metagenome functionality. 


\section{Background}

Ecosystem functioning is mediated by biochemical transformations performed by a community of organisms from every domain of life [1]. In every community, multiple organisms from different taxonomic groups can play similar if not identical roles in ecosystem functionality; the so-called functional redundancy [2]. Functions can be statistically inferred based upon homology to experimentally characterized genes and proteins in specific organisms to find orthologs in other organisms present in a given metagenome. This ortholog annotation, among other annotation tools, can be performed in KEGG Orthology $(\mathrm{KO})[3,4]$, which covers a wide range of functional classes (level 1 of $\mathrm{KO}$ ) comprising cellular processes, environmental information processing, genetic information processing, human diseases, metabolism, organismal system (09150), brite hierarchies and functions not included in the annotation of the two databases pathway or brite. However, the bottleneck of describing metagenome functions is the low number of fully sequenced and annotated genomes as they are mostly limited to those of direct medical or biotechnological interest that have undergone extensive isolation and characterization. Problematically, the vast majority of organisms were not yet studied $[5,6]$ and their annotations are based on their similarity to the genomes of the very few studied model organisms, potentially yielding flawed annotations [7]. Recently, the total functional richness of bacteria [8] and fungi [8,9] was predicted to be millions of KEGG level 3 functions but locally, only a subset of the functions will be available [10]. However, widespread functions present in all metagenomes with abundances depending on the local metagenome density could potentially impact functional indices.

Here, we used a meta-analysis of 20 randomly chosen extant metagenomes each from aerial, aquatic and terrestrial ecosystems representing the full spectrum of metagenome richness in each ecosystem to quantify the widespread functiome. We aimed to analyze the impact of removing widespread functions on the commonly used functional indices of diversity (as Shannon diversity), redundancy and richness. We hypothesize that (i) functionality in aquatic and terrestrial ecosystems will be higher than in aerial metagenomes with generally low biomass [11]. Functionality in both bacteria [8] and fungi $[8,9]$ will be dominated by widespread functions, making up roughly $60 \%$ of the entire functionality which is why we further 
hypothesize that (ii) removing the widespread functiome will result in different estimates of functional indices, potentially resulting in varying differences among the three ecosystems.

\section{Methods}

\section{Data collection and the estimation of functional diversity, redundancy and richness}

The publicly available data was downloaded from the integrated microbial genomes \&

metagenomes (IMG/M) from the Joint Genome Institute (JGI) under https://img.jgi.doe.gov/cgi-bin/m/main.cgi. For each of the three ecosystems aerial, aquatic and terrestrial, the gene counts of KEGG level 3 functions (as "with KO" in the column "Protein coding genes" under "Metagenome Statistics" once a genome was selected) were retrieved from 20 randomly chosen metagenomes representing a full spectrum that covers the whole range of microbial density (indicated by metagenome depth, gene count and number of rRNA copies) on February $14^{\text {th }} 2020$. The distribution of functions among the 20 samples in the three ecosystems was plotted to define the threshold between rare and widespread functiome (Figure 1). Admittedly, the threshold of core functions found in at least 16 of 20 samples was subjectively set as it aligned well with the distribution of functions in aquatic and terrestrial ecosystems. In favor of our threshold of the core functiome were previously determined thresholds of core microbiomes from 16S and ITS2 data that ranged between 30 and 100\% in corals [12] as parallel of core distributions. For aerial metagenomes, there did not seem to exist a clear threshold of core functions indicated by an increase in abundance in aquatic and terrestrial metagenomes, but we aimed to equally analyze the three ecosystems to better compare resulting functional indices. Many of these genes are likely to be housekeeping genes required by almost every organism to maintain basic cellular function. As shown before [8,9], the data structure suggests the presence of more than just rare and widespread functions. Indeed, aquatic metagenomes showed a local maximum of functions in 9 of 20 samples, but here only the core functions were considered. Functional diversity was determined using the Shannon diversity index $H$ according to Equation 1, where $p_{i}$ is the relative abundance of the KEGG level 3 function. Noteworthy, other diversity measures like the Jost indices [13] could be easier to interpret and yield different results but the Shannon index remains the most popular 
in microbial ecology. Functional redundancy was calculated as the average, for all functions in each sample, of how often one KEGG level 3 function was present. Functional richness was determined as the number of different KEGG level 3 functions in each sample.

Eq. 1: $\quad H=-\sum_{i=1}^{k} p_{i} \log \left(p_{i}\right)$

Modelling of the relationship of functional indices to metagenome depth

63

64

65

66

67

First, the relationship between diversity, redundancy and richness to sequencing depth of the metagenome as metagenome depth was fitted to a linear model. While redundancy showed a significant correlation of the linear regression to metagenome depth (Adj. $R^{2}=0.8967, P$-value $<2.2 \mathrm{e}^{-16}$ ), the correlation of diversity to metagenome depth was not significant $\left(\operatorname{Adj} . \mathrm{R}^{2}=\right.$ 0.0042, P-value $=0.268$ ) and both the significance and coefficient of determination were weaker for functional richness $\left(\right.$ Adj. $R^{2}=0.3797$, $P$-value $=9.589 \mathrm{e}^{-8}$ ), with similar results for all functions or when the widespread functions were removed (data shown includes the removal). For diversity and richness, the data was fitted to a saturated (Equation 2) and an unsaturated model (Equation 3) with the critical point estimated by the term $3 A_{f}$ as previously described [14]. The fit of the models was compared by analysis of variance (ANOVA) and Akaike Information Criterion (AIC) [15] with a penalty per parameter set to $k$ equals two.

Eq. 2: Functional richness $=\frac{f_{\max *[\text { Species richness }]}}{A_{f}+[\text { Species richness }]}$

Eq. 3: Function richness $=\frac{f_{\text {max } *[\text { Species richness }]}}{A_{f}+[\text { Species richness }]}+k *[$ Species richness $]$

Here, $f_{\max }$ is the maximum functional richness, $A_{f}$ the accretion rate of functions with an increasing number of species and $k$ the constant of the additive term.

\section{Results}

The abundance of widespread functions in environmental metagenomes

The distribution of KEGG genes over 20 samples showed the highest number of genes in a single sample, totalizing $24.8 \%$ in aerial metagenomes as opposed to $10.9 \%$ in aquatic and $13.2 \%$ in terrestrial metagenomes (Figure 1a). Conversely but only visible in aquatic and terrestrial metagenomes, an increase in KEGG functions was seen when at least 16 of 20 
samples (80\%) were considered, comprising $9.6 \%$ of all KEGG functions in aerial, $24.9 \%$ in aquatic and $25.0 \%$ in terrestrial metagenomes. Noteworthy, another maximum of KEGG functions was visible between $45-60 \%$ of the samples, especially in aquatic ecosystems. In total, aerial, aquatic and terrestrial ecosystems shared 1,201 widespread functions (Figure 1b). Particularly, the widespread KEGG genes found in all ecosystems were affiliated with metabolic pathways (427), biosynthesis of secondary metabolites (217), biosynthesis of antibiotics (154), microbial metabolism in diverse environments (89) and carbon metabolism (89) as the five most abundant pathways. On the level of modules, the widespread KEGG genes included the citrate cycle (16), NADH:quinone oxidoreductase in prokaryotes (15), reductive citrate cycle (14), 2-oxoglutarate $\rightarrow$ oxaloacetate (13) and glycolysis (12) as the top five most abundant modules (a detailed list of the widespread functions can be found in the supplementary information, Table S1). Only 6 widespread functions were solely found in aerial ecosystems as opposed to 1,021 in aquatic and 279 in terrestrial metagenomes. Aerial and aquatic ecosystems shared 10, aerial and terrestrial metagenomes shared 22, and aquatic and terrestrial ecosystems shared 1,954 widespread functions.

\section{The relationship of functional indices and metagenome depth}

When the functional indices of all functions were compared to the functions remaining after the removal of widespread functions, statistically similar functional diversities across the three metagenomes were observed (Tukey HSD, P-value > 0.05) [16] (Figure 2). However, the removal of widespread functions resulted in generally lower values and a higher variation within each ecosystem. Similarly, the pattern of significantly (P-value $<0.05$, HSD-test) lower functional redundancy in aerial metagenomes was found whether all functions were used or if the widespread functions were removed. However, even though the values were generally lower, they also showed lower variation. Lastly, functional richness was significantly (Tukey HSD, P-value < 0.05) higher in aquatic metagenomes compared to terrestrial and aerial ecosystems. With the removal of widespread functions, the three ecosystems showed similar functional richness, which was generally lower and had a higher variation. Functional diversity of all functions showed an asymptote that tends to towards 7.6, indicated by the better fit to the saturated model (Figure 3a) while functional redundancy showed a significant positive 
113 linear correlation to metagenome depth (Figure 3b). Otherwise, the correlation between 114 functional richness and metagenome depth was best described by an unsaturated model 115 (Figure 3c). Admittedly, the difference in AIC of the saturated and unsaturated model was lower $(0.7 \%)$ for richness as compared to diversity (AIC-difference $=1.7 \%$ ), which could mean that the 117 evenness changed. The relationships were of identical nature when the widespread functions 118 were removed but a higher variation in both functional diversity (Figure 4a) and richness 119 (Figure 4c) resulted in higher AIC values and less significant parameters with higher standard errors. On the contrary, functional redundancy showed a higher coefficient of determination 121 with the removal of widespread functions albeit with a much slighter slope and overall lower 122 values (Figure 4b). Noteworthy, due to the wide range of metagenome depths that are 123 dominated by low depth metagenomes near or equal to zero in both redundancy and richness, 124 both slopes and correlations of the variables are likely skewed towards the high depth metagenomes. However, the important aspect of the correlations are the higher variations of functional diversity and functional richness and a reduction of functional redundancy.

\section{Discussion}

Consistent with our first hypothesis, both functional redundancy and richness were significantly ( $P$-value $<0.05)$ higher in aquatic and terrestrial metagenomes as compared to aerial metagenomes. Otherwise, functional diversities showed similar values in the three ecosystems. In aerial metagenomes, low redundancy and richness may be a direct consequence of low metagenomic density, as depicted by a sequencing depth several orders of magnitude lower than for their aquatic and terrestrial counterparts. Noteworthy, a sufficient sequencing depth is key to cover all organisms and their functions in each sample and could result in lower functional indices values but with the data at hand, it is hard to ascertain. Even in environments of low functionality, the functional diversity still reaches a similar maximum at a Shannon diversity of 7.6 \pm 0.1 . Because diversity is based on both richness and evenness, a higher evenness could counteract a low richness to yield a comparable diversity. This derives from the structure of functions and their distribution in sequenced metagenomes that seemed to be similar in every metagenome if a certain threshold of metagenome density is reached. In our 
141 case, the threshold was with a metagenome depth of $100,000,000$ bases. In comparison, the 142 size of the fungal genomes of Aspergillus nidulans and Saccharomyces cerevisiae were reported 143 to be $30,000,000$ [17] and $12,100,000$ base pairs (from the Saccharomyces genome database), respectively. Even though bacterial genomes tend to be an order of magnitude smaller, i.e. 4,600,000 base pairs of Escherichia coli [18], and other organisms such as viruses have even smaller genomes but can contribute to the functionality, a metagenome of $100,000,000$ bases will likely comprise of only a few different organisms. With that in mind, we assume that the maximum potential of functional diversity is reached in every energy or nutrient unlimited environment, particularly in aquatic and terrestrial ecosystems. Both functional redundancy and richness increased with increasing metagenome depth, implying unlimited potential of functional diversity with metagenome depth. Adding more organisms will not only add more rare functions but also increase the chances of the addition of already present widespread (or rare) functions $[8,9]$ that, in turn, will increase both functional redundancy and richness. However, the local metagenome will be limited by space and resource availability as well as constraining environmental conditions which is why unlimited functional redundancy and richness are only theoretically possible. The largest metagenome in our meta-analysis was of $6,556,162,052$ bases, representing thousands of different organisms inhabiting the marine sediment from White Oak River estuary in North Carolina, and could indicate the maximum capacity of functional diversity.

The removal of widespread functions logically resulted in lower values for all indices with higher variation in diversity and richness but lower variation in redundancy. The Shannon index takes richness and evenness into account, which is why removing widespread functions increased evenness more than it reduces richness, resulting in a higher diversity. In line with our second hypothesis, the significant differences in functional richness vanished with the removal of widespread functions, indicating that most functions especially in microbe-rich environments are indeed widespread. Further, similar functional richness of non-widespread functions indicated that the total functionality in aerial, aquatic and terrestrial metagenomes is very similar despite the different microbial density. Otherwise, functional diversity (similar between the three ecosystems) and redundancy (significantly lower in aerial metagenomes) 
170 still showed the same trends. Only functional redundancy was able to capture the difference 171 between aerial, aquatic and terrestrial ecosystems after the removal of widespread functions 172 because those were presumably highly redundant functions. However, greater variation in both 173 diversity and richness can be advantageous to compare microbe-rich environments. 174 Particularly, functional diversity ranged from 6.19 to 7.64 after the removal of widespread 175 functions, which represents a two-fold increase in range compared to all functions (7.37-8.10). 176 The scale of sequencing depths between habitats varied greatly but we aimed to depict a 177 representation of each of those habitats that are publicly available rather than normalizing the 178 amount of sequences. However, the outcome could be affected as there is a higher probability 179 of sequencing already abundant genes or functions by chance as opposed to rarer ones.

\section{Conclusion}

Altogether, we unveil the weight of widespread functions in statistical analyses and quantify their abundance. The removal of widespread functions resulted in a higher quality of functional diversity, redundancy and richness, and will make possible the comparison of microbe-rich environments that would otherwise have a similar distribution. Admittedly, our estimates are based on a meta-analysis of 20 randomly chosen metagenomes that represent a snapshot of all possibilities but given the low number of aerial metagenomes $(n=108)$, it seemed to be the most feasible and unbiased approach. Moving forward, we recommend the removal of widespread functions, i.e. by using the functions provided in Table S1, to analyze metagenome functionality as we demonstrated their tremendous impact of functional indices. A core functiome needs to be defined similar to the core microbiome where widespread/generalist functions are done by all or most and rare/specialist functions are done by only a few or in rare circumstances [19]. Other than the similarity-based assessment of functions, the production of knock-ins on a large scale by adding whole contigs and sequencing the mutant inserts of potentially interesting phenotypes represents an interesting approach suitable for unveiling yet unknown functions [20]. 
197 Ethics approval and consent to participate

198 Not applicable

199 Consent for publication

200 Not applicable

201 Availability of data and material

202 The data is publicly available at the integrated microbial genomes \& metagenomes (IMG/M)

203 from the Joint Genome Institute (JGI) under https://img.jgi.doe.gov/cgi-bin/m/main.cgi

204 Competing interests

205 The authors declare no competing interests.

206 Funding

207 This work was supported by the Czech Science Foundation (20-02022Y).

208 Author's contribution

209 RS and SPC designed the study. RS analyzed the data. RS and SPC wrote and revised the 210 manuscript. All authors read and approved the final manuscript.

211 Acknowledgements

212 Not applicable

\section{References}

214 1. Woese CR, Kandler O, Wheelis ML. Towards a natural system of organisms: proposal for the 215 domains Archaea, Bacteria, and Eucarya. Proc Natl Acad Sci. 1990;

216 2. Hubbell SP. Neutral theory in community ecology and the hypothesis of functional 217 equivalence. Funct Ecol. 2005;

218 3. Kanehisa M, Sato Y, Kawashima M, Furumichi M, Tanabe M. KEGG as a reference resource for 
gene and protein annotation. Nucleic Acids Res. 2016;

220

4. Kanehisa M, Sato Y, Morishima K. BlastKOALA and GhostKOALA: KEGG Tools for Functional Characterization of Genome and Metagenome Sequences. J. Mol. Biol. 2016.

5. Pham VHT, Kim J. Cultivation of unculturable soil bacteria. Trends Biotechnol. 2012.

223

6. Martiny AC. High proportions of bacteria are culturable across major biomes. ISME J. 2019;

7. Salzberg SL. Next-generation genome annotation: We still struggle to get it right. Genome 225 Biol. 2019.

8. Starke R, Capek P, Morais D, Callister SJ, Jehmlich N. The total microbiome functions in bacteria and fungi. J Proteomics. 2020;

9. Starke R, Capek P, Morais D, Jehmlich N, Baldrian P. Explorative Meta-Analysis of 377 Extant Fungal Genomes Predicted a Total Mycobiome Functionality of 42.4 Million KEGG Functions. Front Microbiol. 2020;11:143.

10. Starke R. Meta-analysis of 139 extant Tara ocean metagenomes to unveil the relationship

232 between taxonomy and functionality in prokaryotes inhabiting aquatic ecosystems. bioRxiv. 2332020 ;

11. Lozupone CA, Knight R. Global patterns in bacterial diversity. Proc Natl Acad Sci U S A. 2007;

12. Hernandez-Agreda A, Gates RD, Ainsworth TD. Defining the Core Microbiome in Corals' Microbial Soup. Trends Microbiol. 2017.

237 13. Chao A, Chiu CH, Jost L. Phylogenetic diversity measures based on Hill numbers. Philos Trans 238 R Soc B Biol Sci. 2010;

239 14. Čapek $P$, Kotas $P$, Manzoni S, Šantrůčková H. Drivers of phosphorus limitation across soil 240 microbial communities. Funct Ecol. 2016;

241 15. Bertrand P V., Sakamoto Y, Ishiguro M, Kitagawa G. Akaike Information Criterion Statistics. J 242 R Stat Soc Ser A (Statistics Soc. 2006;

243 16. Tukey JW. Comparing Individual Means in the Analysis of Variance. Biometrics. 1949; 
244 17. Galagan JE, Calvo SE, Cuomo C, Ma LJ, Wortman JR, Batzoglou S, et al. Sequencing of

245 Aspergillus nidulans and comparative analysis with A. fumigatus and A. oryzae. Nature. 2005;

246 18. Blattner FR, Plunkett G, Bloch CA, Perna NT, Burland V, Riley M, et al. The complete genome 247 sequence of Escherichia coli K-12. Science (80-. ). 1997.

248 19. Saavedra-Lavoie J, de la Porte A, Piché-Choquette S, Guertin C, Constant P. Biological h2 and 249 co oxidation activities are sensitive to compositional change of soil microbial communities. Can 250 J Microbiol. 2020;

251 20. Brahami A, Castonguay A, Déziel É. Novel 'Bacteriospray' Method Facilitates the Functional 252 Screening of Metagenomic Libraries for Antimicrobial Activity. Methods Protoc. 2019; 
254 Figure and figure legends
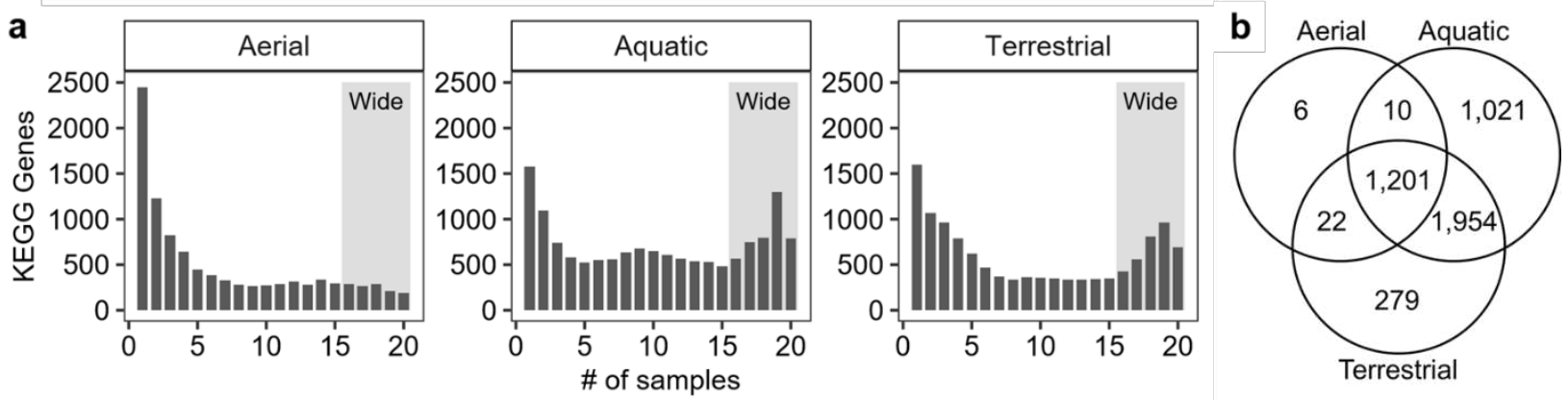

255

Figure 1: The number of genes with KEGG level 3 functions found in subsets of the 20 aerial,

257 aquatic and terrestrial metagenomes with their widespread functiome in at least 16 of 20

258 samples indicated in grey (a) and the share of widespread functions between the three 259 ecosystems (b). 

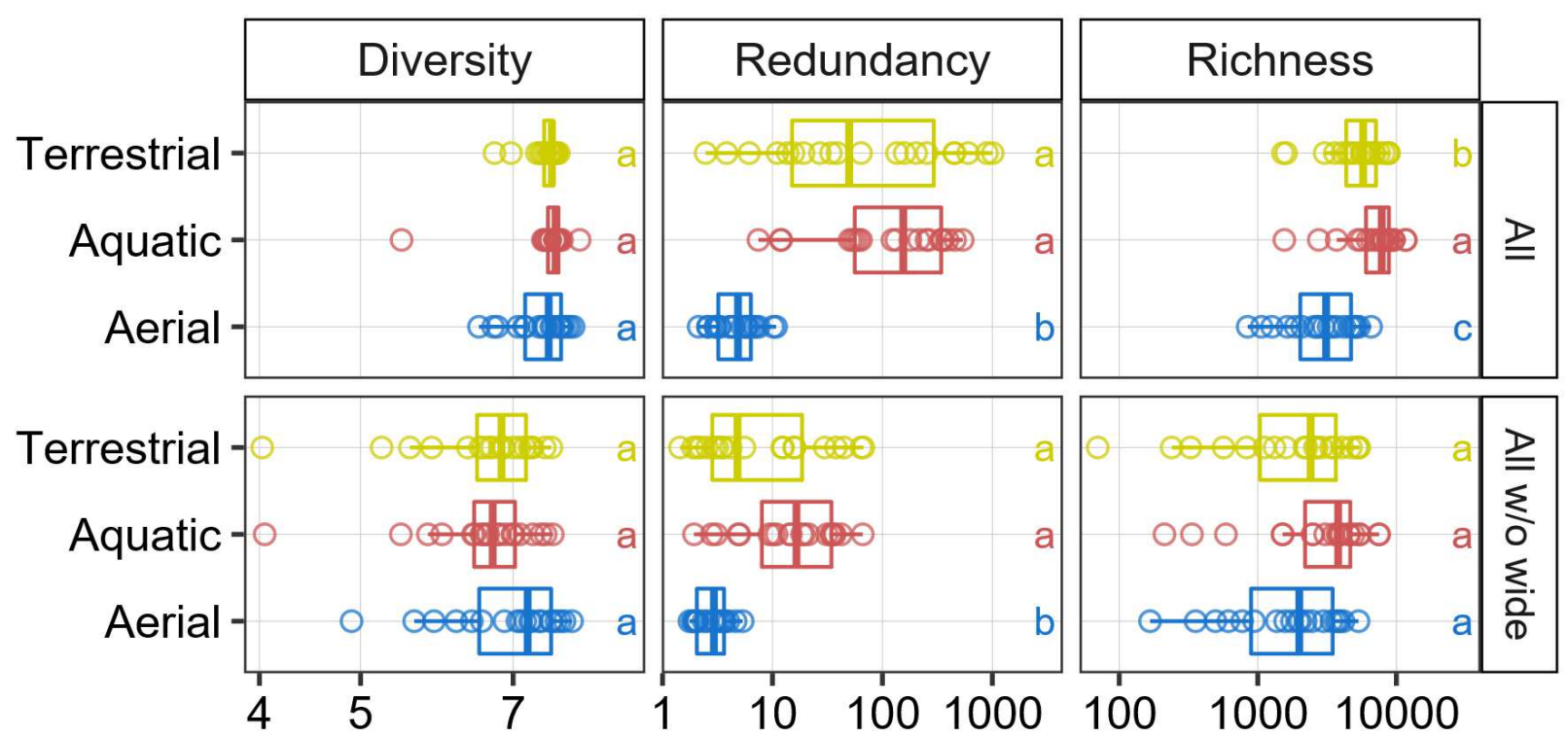

262 metagenomes of the whole range of microbial density when all functions are considered (All) or

263 when the widespread functions are removed (All w/o wide) (a). Groups followed by the same

264 letter are not significantly different according to the HSD test (P-value > 0.05). 

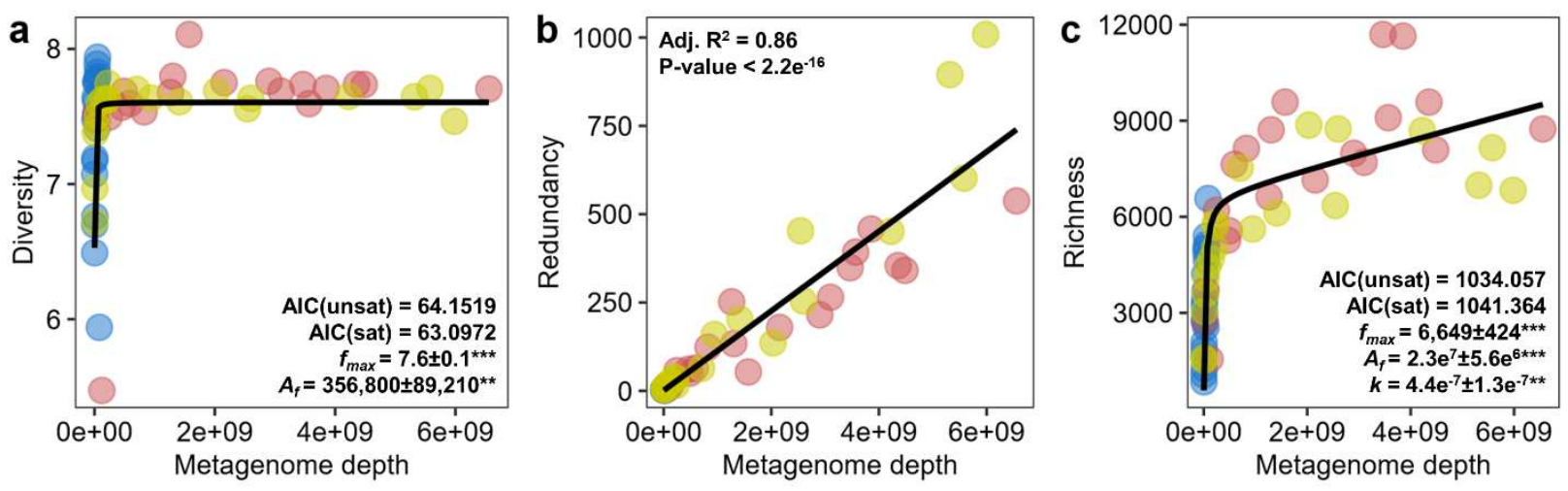

266 Figure 3: The saturated relationship of functional diversity, linear relationship of functional

267 redundancy and unsaturated relationship of functional richness to metagenome depth when all 268 functions are considered. Aerial samples are shown in blue, aquatic in red and terrestrial in 269 yellow. Asterisks indicate the significance of individual parameters in saturated and unsaturated models $(*=$ P-value $<0.05, * *=$ P-value $<0.01$ and $* * *=$-value $<0.001)$. 

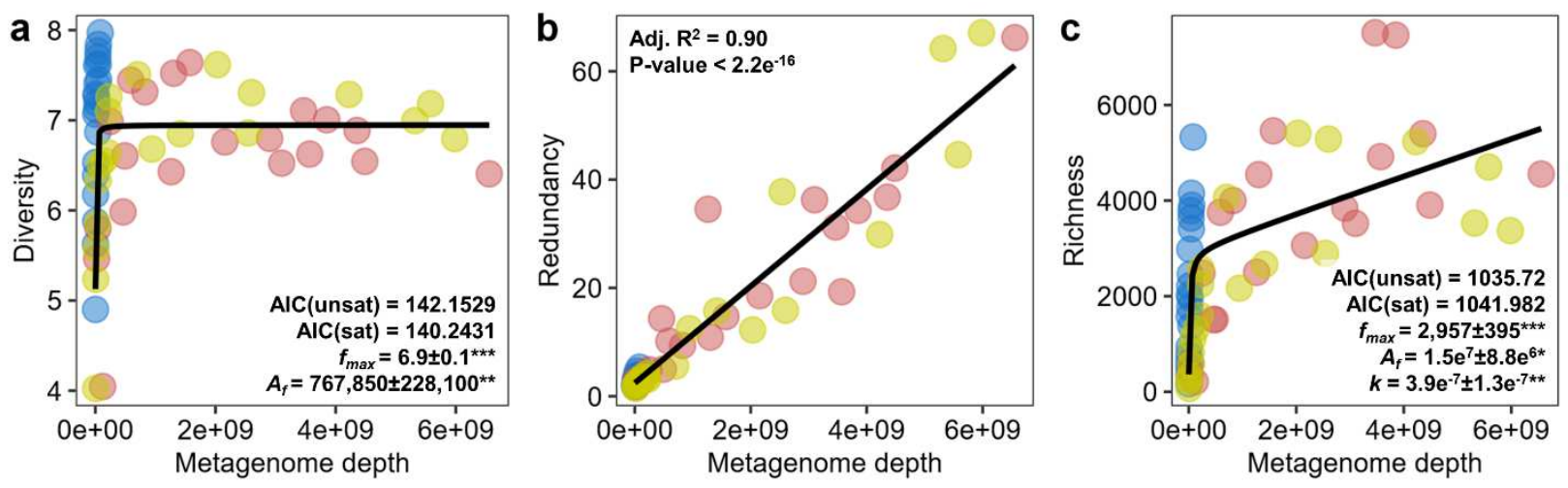

272 Figure 4: The saturated relationship of functional diversity, linear relationship of functional

273 redundancy, and unsaturated relationship of functional richness to metagenome depth when

274 the widespread functions are removed. Aerial samples are shown in blue, aquatic in red and

275 terrestrial in yellow. Asterisks indicate the significance of individual parameters in saturated

276 and unsaturated models ( $*=\mathrm{P}$-value $<0.05, * *=\mathrm{P}$-value $<0.01$ and $* * *=\mathrm{P}$-value $<0.001)$. 


\section{Figures}
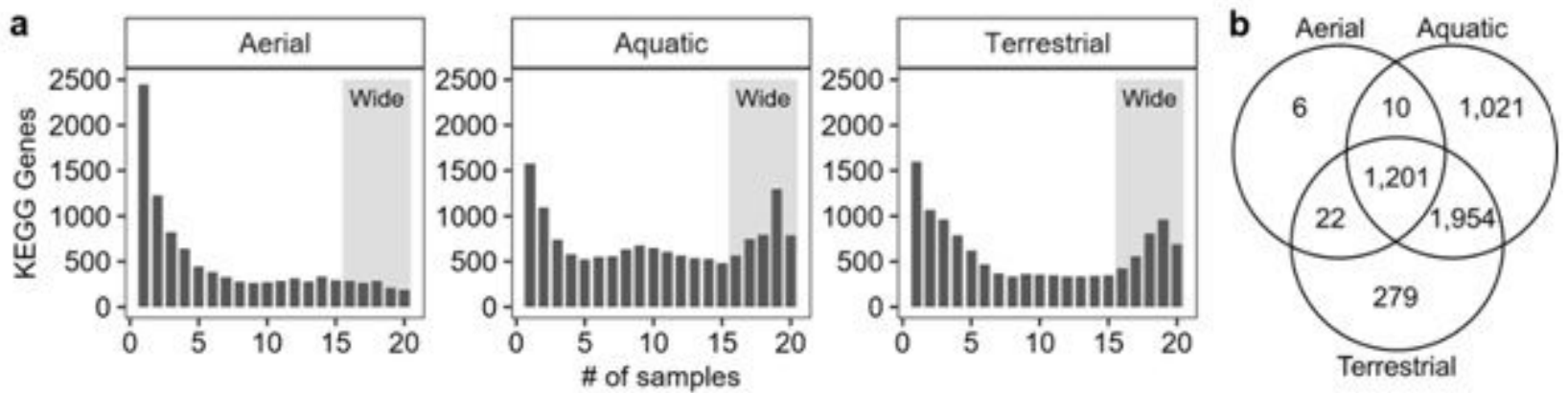

Figure 1

The number of genes with KEGG level 3 functions found in subsets of the 20 aerial, aquatic and terrestrial metagenomes with their wide spread functiome in at least 16 of 20 samples indicated in grey (a) and the share of widespread functions between the three ecosystems (b).

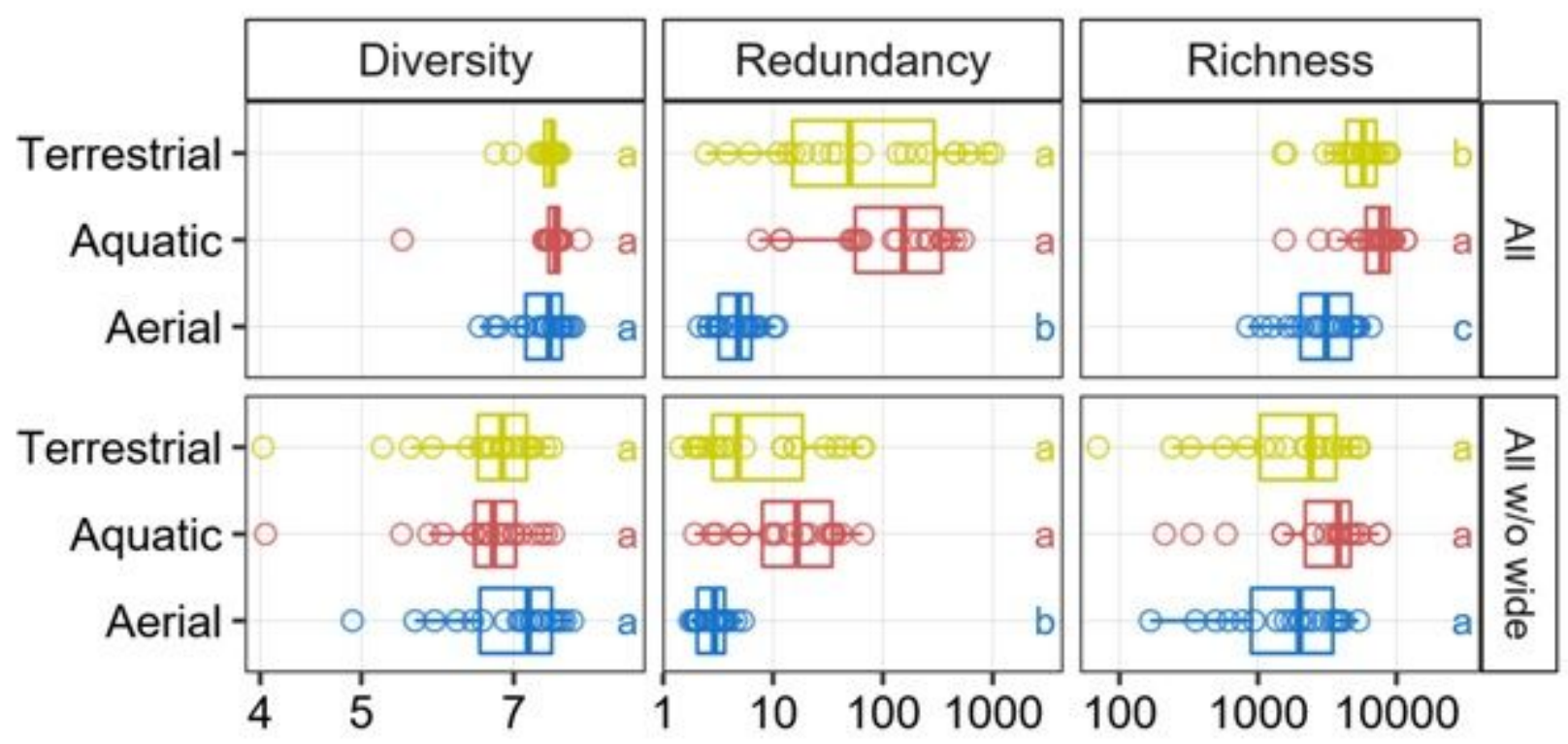

Figure 2

Functional diversity, redundancy and richness in aerial, aquatic and terrestrial metagenomes of the whole range of microbial density when all functions are considered (All)or when the widespread functions are removed (All w/o wide) (a). Groups followed by the same letter are not significantly different according to the HSD test $(P$-value $>0.05)$. 

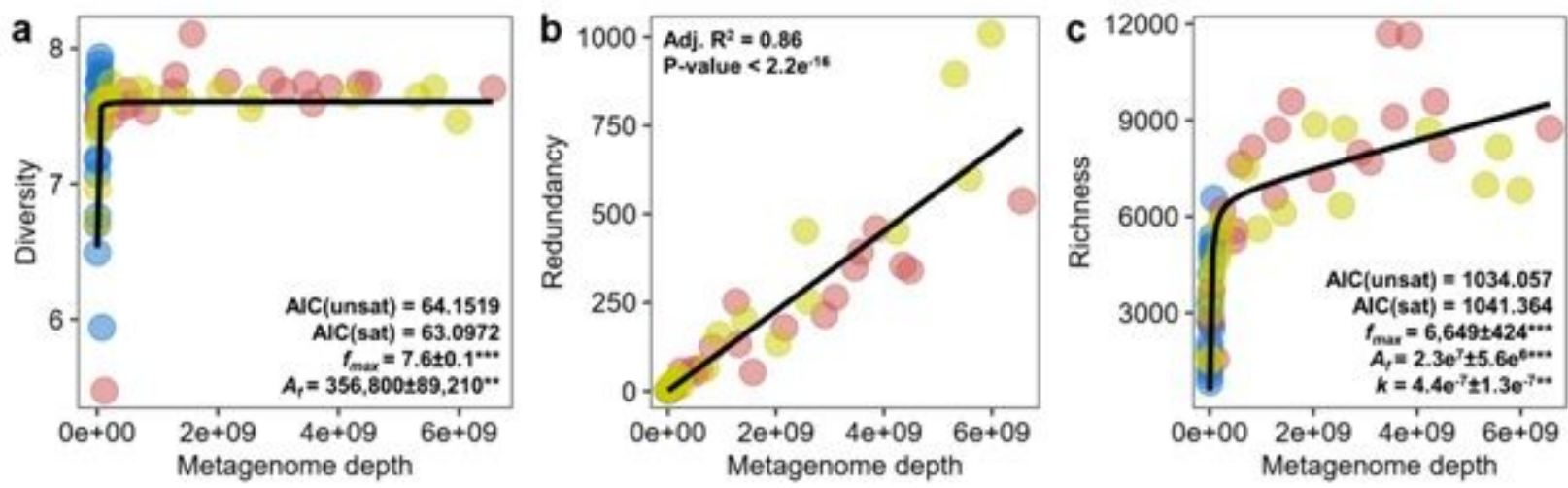

Figure 3

The saturated relationship of functional diversity, linear relationship of functional redundancy and unsaturated relationship of functional richness to metagenome depth when all functions are considered. Aerial samples are shown in blue, aquatic in red and terrestrial in yellow. Asterisks indicate the significance of individual parameters in saturated and unsaturated models $\left({ }^{*}=\mathrm{P}\right.$-value $<0.05,{ }^{*}=\mathrm{P}$ value $<0.01$ and $* * *=$ P-value $<0.001)$.
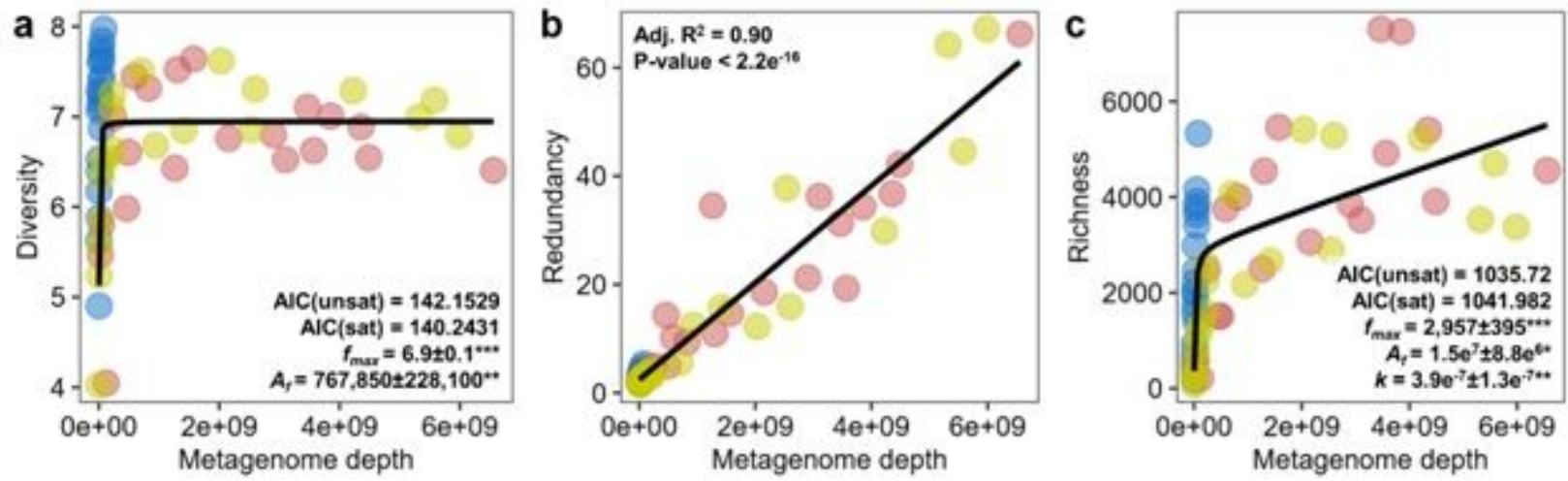

Figure 4

The saturated relationship of functional diversity, linear relationship of functional redundancy, and unsaturated relationship of functional richness to metagenome depth when the widespread functions are removed. Aerial samples are shown in blue, aquatic in red and terrestrial in yellow. Asterisks indicate the significance of individual parameters in saturated and unsaturated models $(*=P$-value $<0.05, * *=P$ value $<0.01$ and $* * *=P$-value $<0.001)$.

\section{Supplementary Files}

This is a list of supplementary files associated with this preprint. Click to download.

- TableS1.xlsx 